\title{
Glyoxal's impact on dry ammonium salts: fast and reversible surface aerosol browning
}

\section{Supplemental Information}

\author{
David O. De Haan, * 1 Lelia N. Hawkins, 2 Kevin Jansen,3 Hannah G. Welsh, 2 Raunak \\ Pednekar,2,5 Alexia de Loera,1 Natalie G. Jimenez,1 Margaret A. Tolbert,3 Mathieu Cazaunau,4 \\ Aline Gratien,4 Antonin Bergé,4 Edouard Pangui, 4 Paola Formenti,4 Jean-François Doussin 4
}

1Department of Chemistry and Biochemistry, University of San Diego, 5998 Alcala Park, San Diego CA 92110 USA

2Department of Chemistry, Harvey Mudd College, 301 Platt Blvd, Claremont CA 91711 USA ...

3Department of Chemistry / Cooperative Institute for Research in Environmental Sciences, University of Colorado, Boulder, CO 80309 USA

4Laboratoire Interuniversitaire des Systèmes Atmosphériques (LISA), UMR7583, CNRS, Université Paris-Est-Créteil (UPEC) et Université Paris Diderot (UPD), Institut Pierre Simon Laplace (IPSL), Créteil, France

5 Deceased

*Corresponding_Author, ddehaan@sandiego.edu 


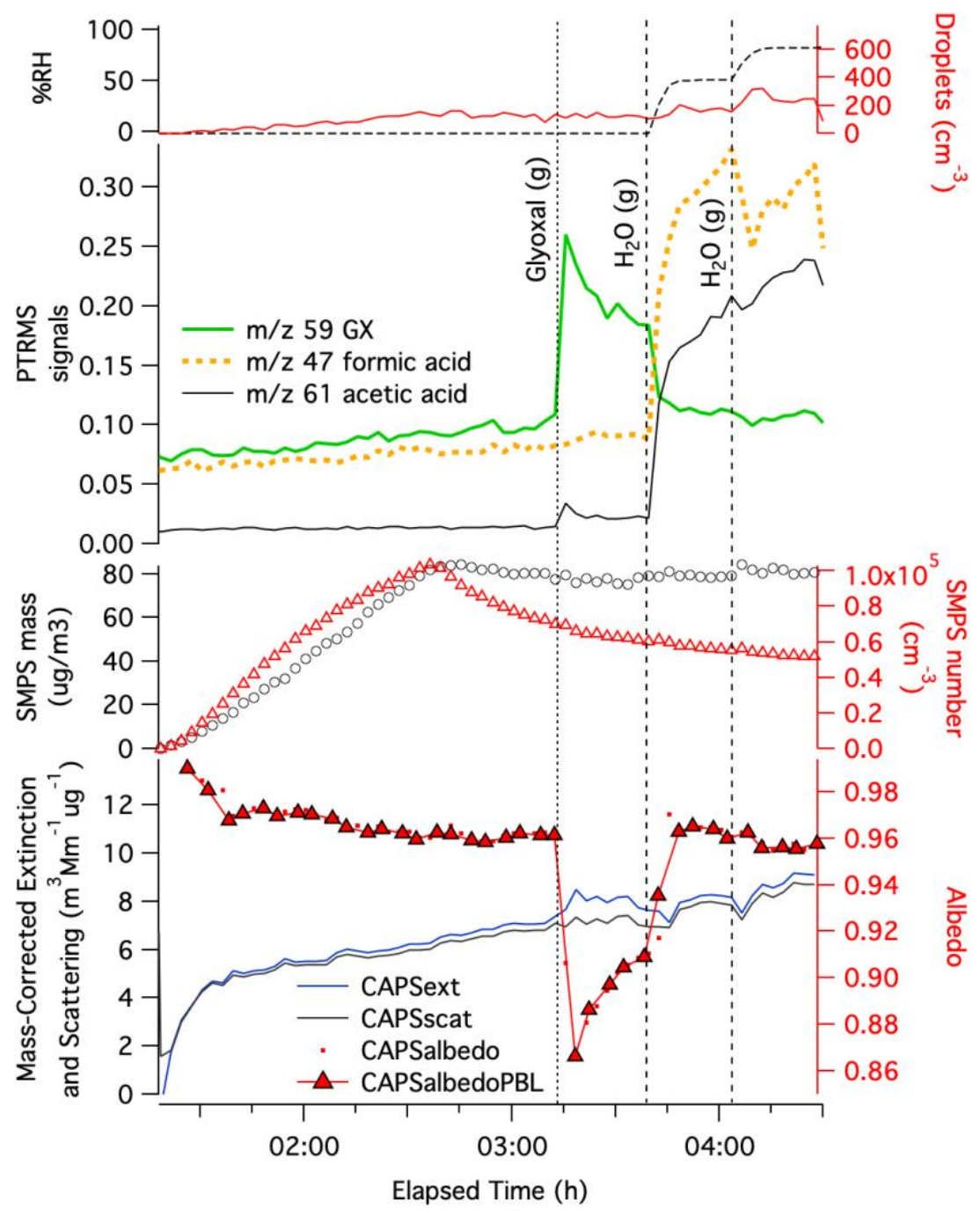

Figure S1: Ammonium sulfate / glycine seed experiment 2. Top: chamber RH and cloud droplet counts, traces color-coded to axes. Middle top: dilution- and water-corrected PTR-MS traces are shown for gas-phase glyoxal $(\mathrm{m} / \mathrm{z}=59$, green line), formic acid $(\mathrm{m} / \mathrm{z} 47$, yellow dotted line), and acetic acid ( $\mathrm{m} / \mathrm{z}$ 61, black line). Middle bottom: dilution-corrected SMPS number density and particulate mass (assuming aerosol density $=1$ ) are shown next, with increasing number density indicating internally-mixed AS/glycine aerosol addition at start of experiment. Bottom: massnormalized 2-min averaged CAPS extinction (blue line), scattering (black line), single-scattering albedo (red dots), and albedo values calculated from data immediately following instrument baseline (red triangles), all measured at $450 \mathrm{~nm}$. Additions of $0.25 \mathrm{ppm}$ glyoxal gas (vertical dotted line), water vapor addition and 2 cloud events (dashed lines), and start of chamber illumination (red line) are labeled. 


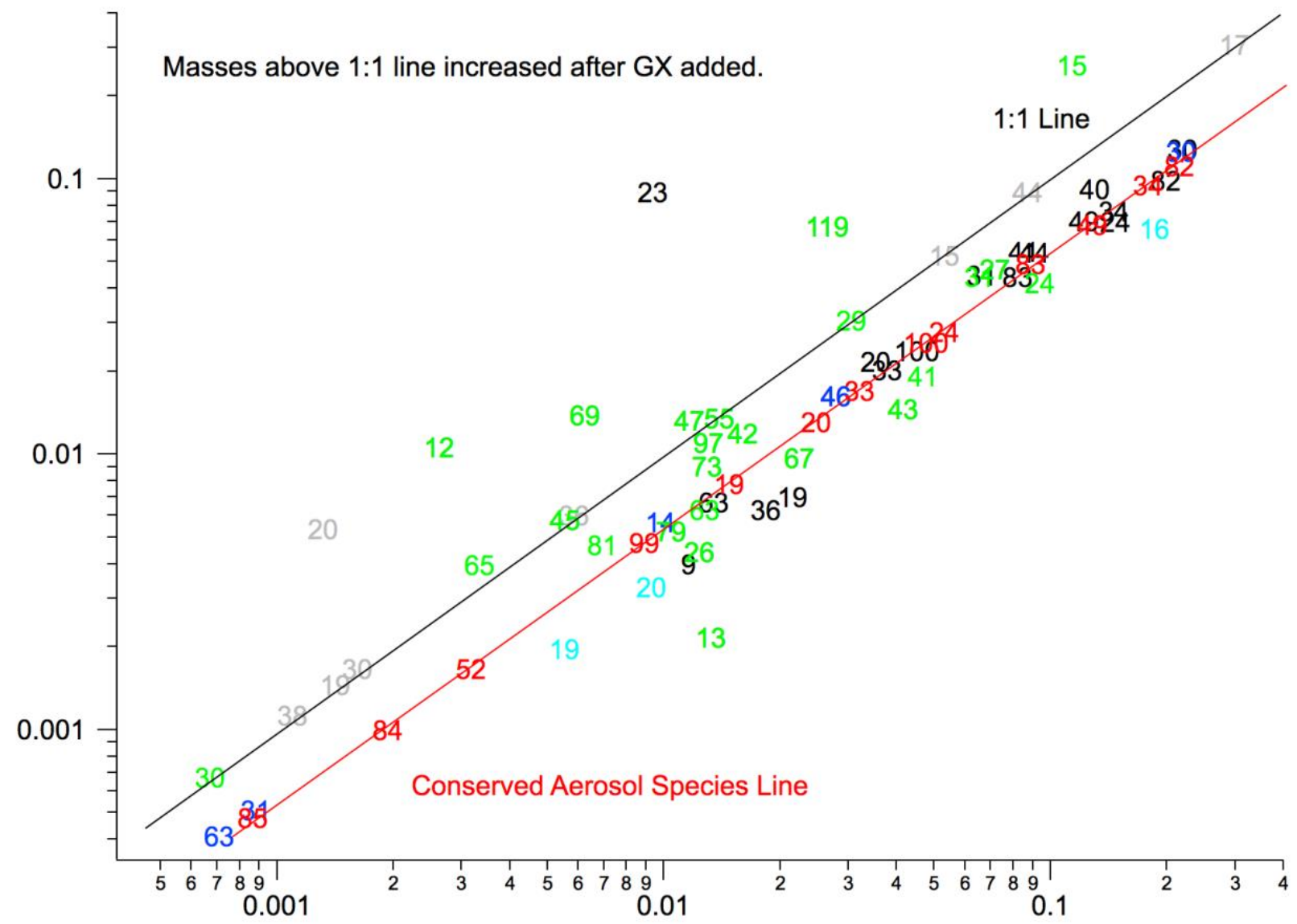

Figure S2: Experiment 3 AMS data summary: dry AS aerosol +40 ppb glyoxal gas. Overall, aerosol concentrations decreased due to wall losses during the experiment, resulting in ions corresponding to conserved aerosol species falling on the red line with a slope of 1 , slightly below the 1:1 line. However, certain ions increased upon GX addition and appear above the 1:1 line: $m / z 15$ ( $\mathrm{CH}_{3}$ or NH fragment), $m / z 23(\mathrm{Na}), m / z 119$ (imidazole carboxaldehyde, sodium adduct 97+22), $m / z 69$ (imidazole), and $m / z 47$ (formic acid). Ions associated with water (shown in light blue at $m / z 16,19$, and 20) declined slightly upon glyoxal addition, appearing below the red line. 


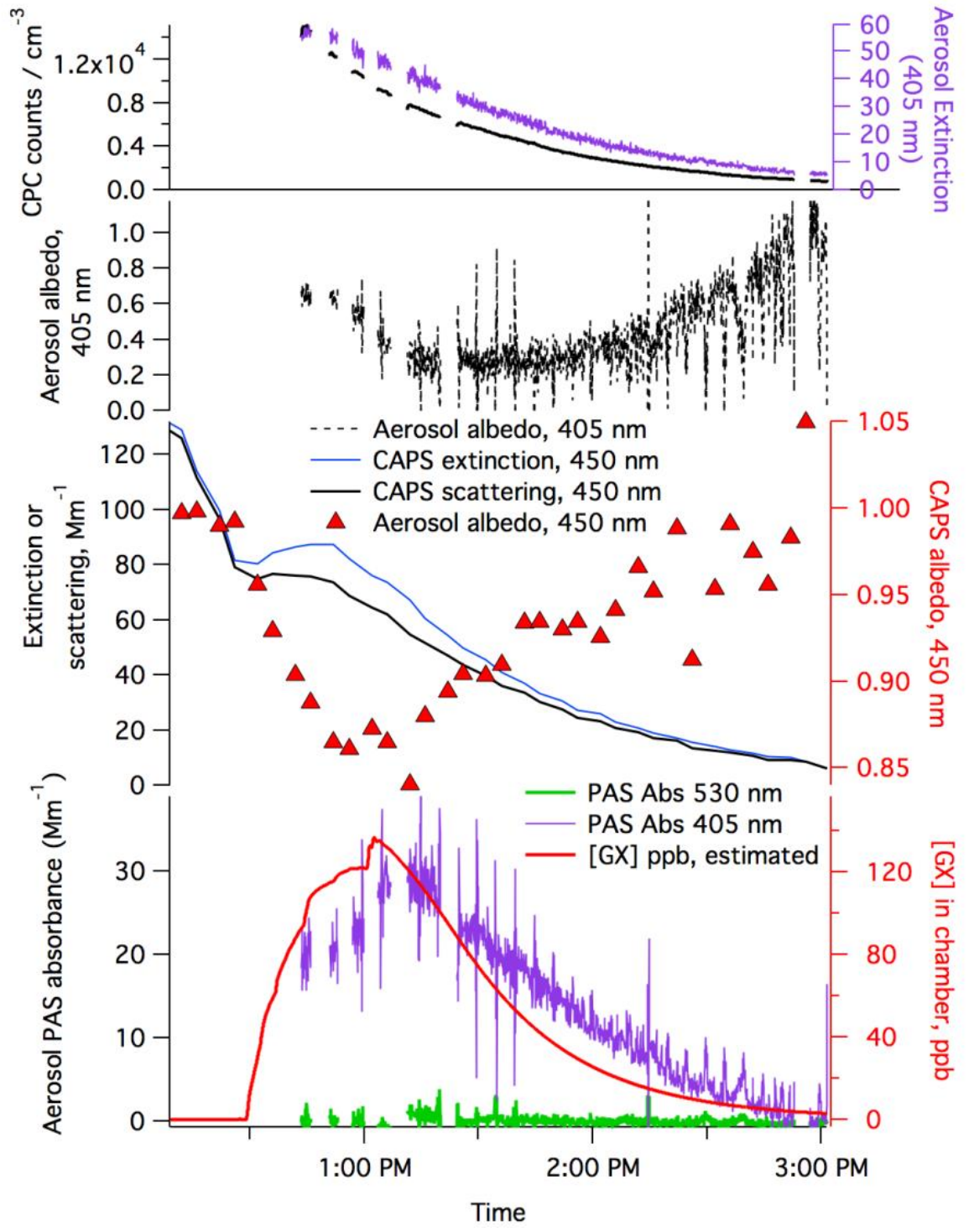

Figure S3: Gradual glyoxal addition experiment 8 on dry MeAS aerosol in small Tedlar chamber.

Top: Aerosol CPC counts (black) and CRD extinction at $405 \mathrm{~nm}$ (blue). 2nd panel: Aerosol albedo at $405 \mathrm{~nm}$ (black dashed line), calculated from PAS and CRD data. 3rd panel: aerosol extinction (blue), scattering (black), and albedo (red triangles), all measured at $450 \mathrm{~nm}$ by CAPS-ssa. Bottom: Aerosol absorbance measured by PAS at $405 \mathrm{~nm}$ (blue) and $530 \mathrm{~nm}$ (green), and estimated glyoxal concentrations in the chamber (red, calculated using wall loss rate $=6.7 \times 10-4$ S-1). Right axes are color-coded. 


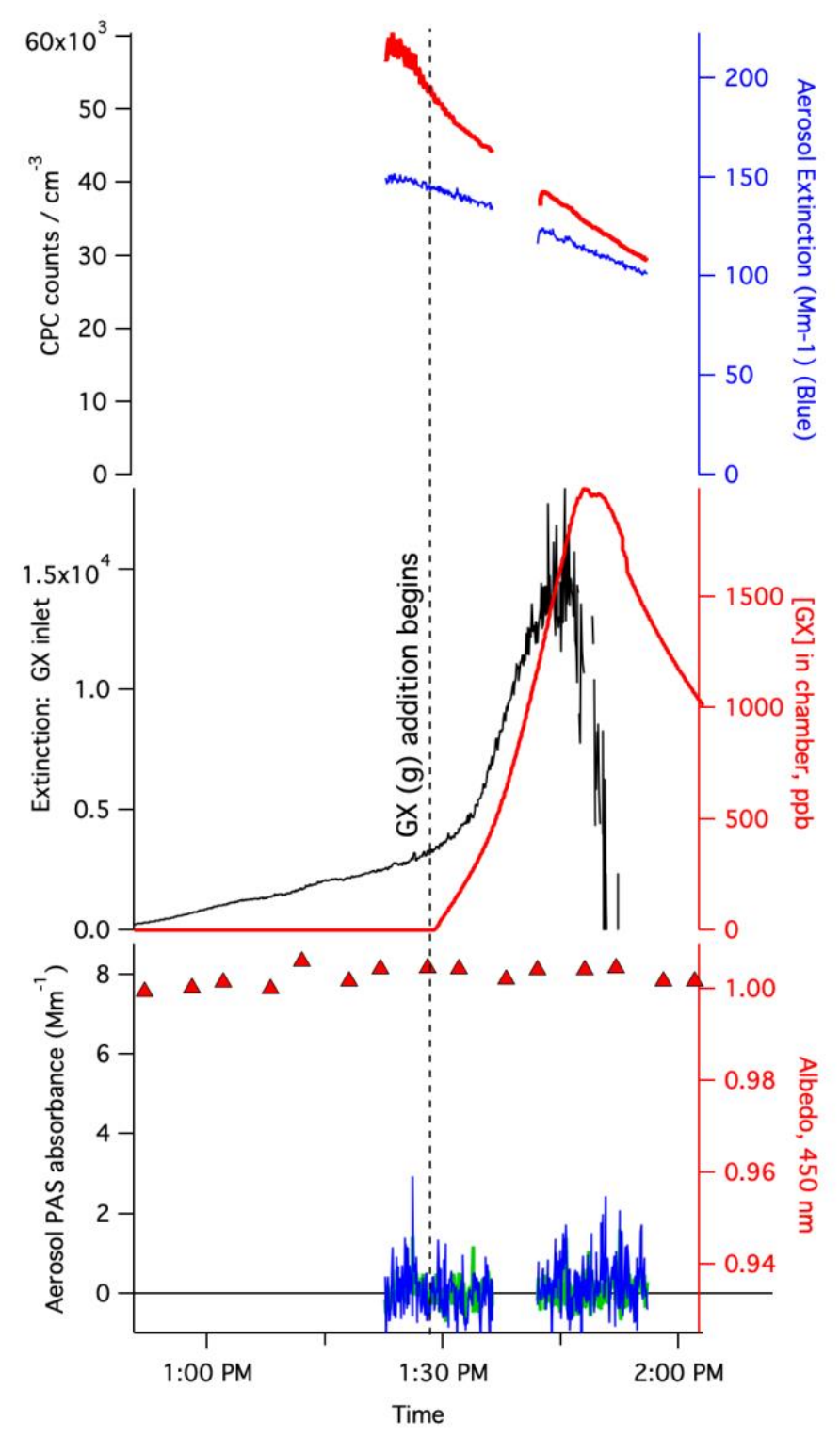

Figure S4: Gradual glyoxal addition experiment 9 on dry sodium sulfate $\left(\mathrm{Na}_{2} \mathrm{SO}_{4}\right)$ aerosol in small Tedlar chamber. Top: Aerosol CPC counts (red) and CRD extinction at $405 \mathrm{~nm}$ (blue). Middle: CRD extinction at $405 \mathrm{~nm}$ due to glyoxal, measured at glyoxal inlet (black), and estimated glyoxal concentrations in the chamber (red, calculated using wall loss rate $=6.7 \times 10-4 \mathrm{~S}-1$ ). These chamber concentrations (and the glyoxal wall loss rate) were confirmed by measuring glyoxal concentrations by CRD extinction at $405 \mathrm{~nm}$ at the chamber outlet at $3 \mathrm{pm}$. Bottom: CAPS-ssa albedo at $450 \mathrm{~nm}$ (red triangles), and aerosol absorbance measured by PAS at $405 \mathrm{~nm}$ (blue) and $530 \mathrm{~nm}$ (green). 


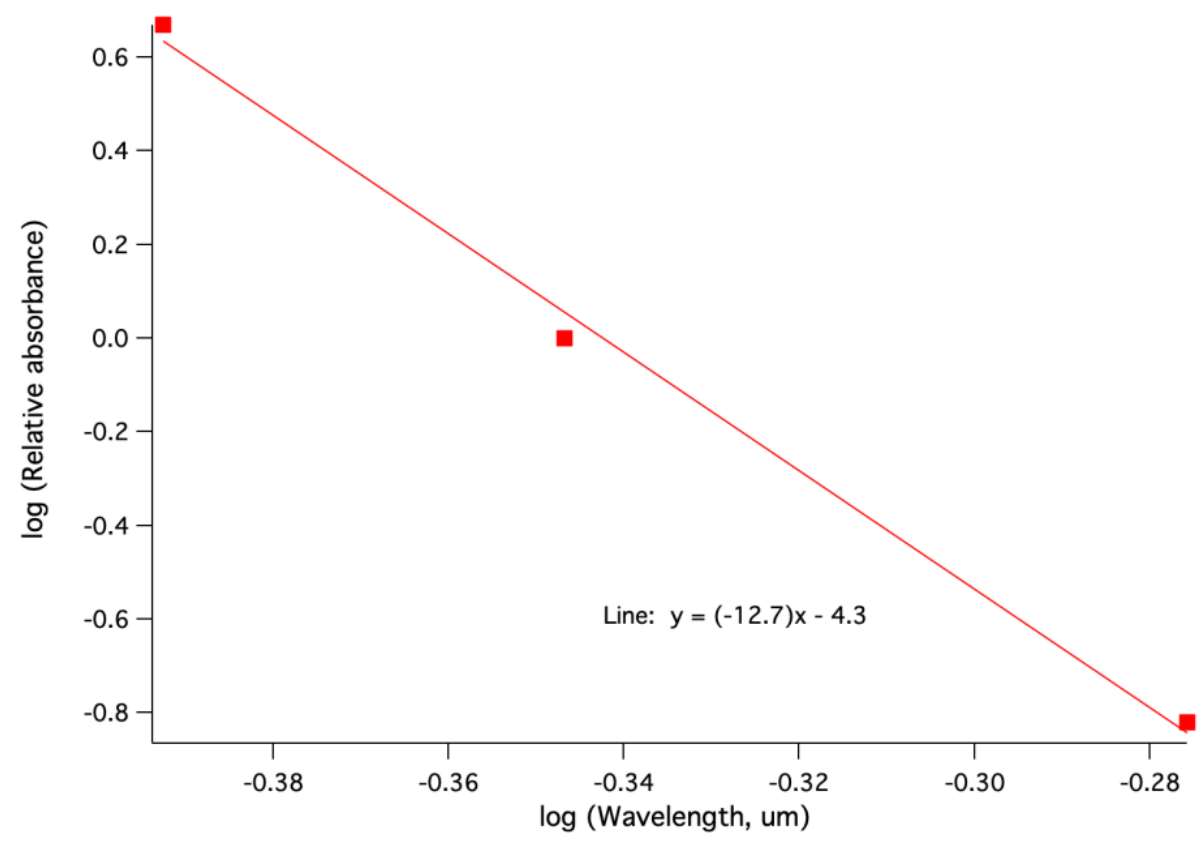

Figure S5: Calculation of angstrom absorption coefficient from normalized aerosol optical data recorded at 405,450 , and $530 \mathrm{~nm}$ at $1: 11 \mathrm{pm}$ in experiment 8 (140 ppb glyoxal + dry methylammonium sulfate aerosol). Slope of line is Ångstrom absorption coefficient. 


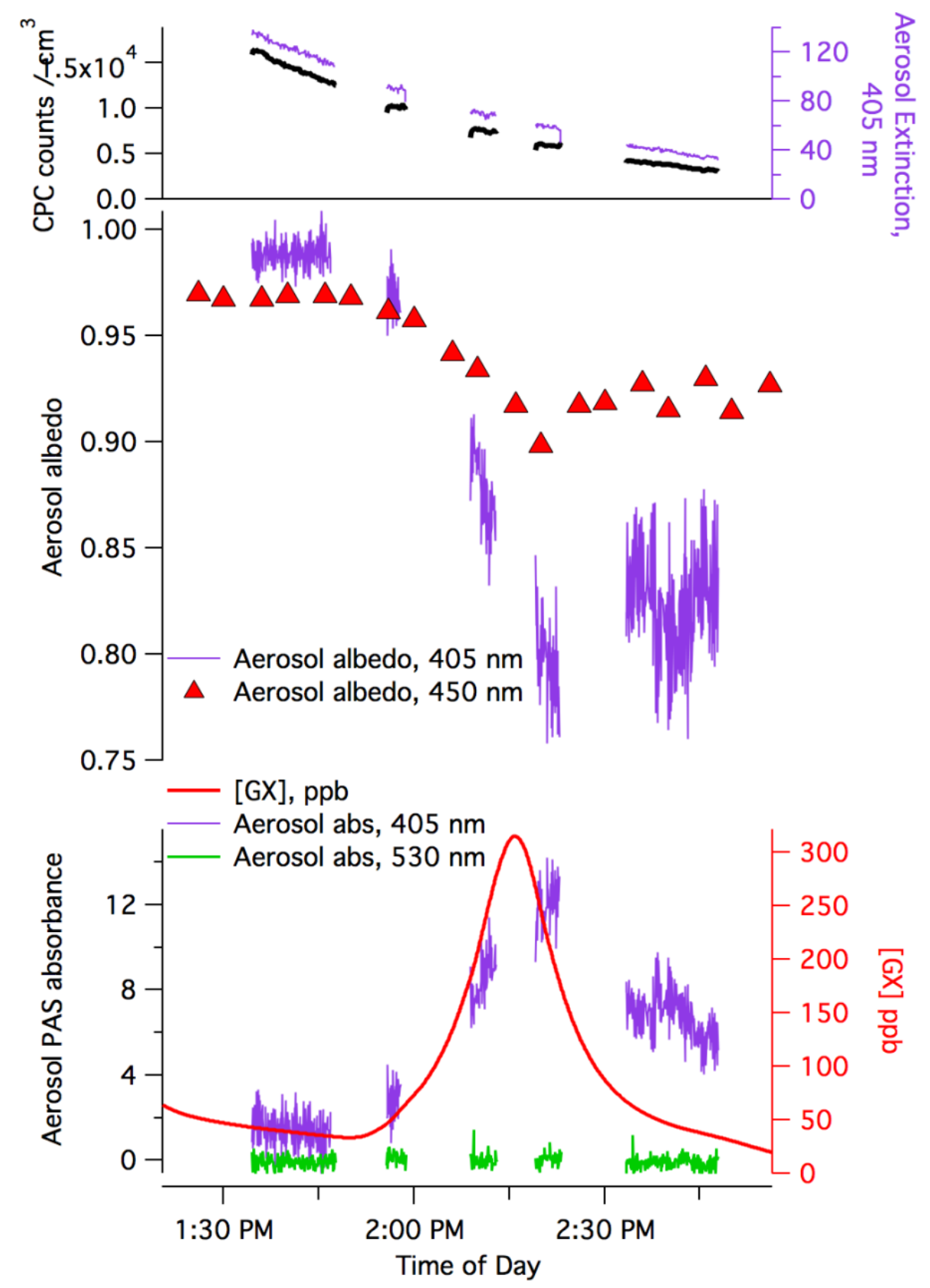

Figure S6: Gradual glyoxal addition experiment 4 on dry ammonium sulfate (AS) aerosol in small Tedlar chamber. Top: Aerosol CPC counts (black) and CRD extinction at $405 \mathrm{~nm}$ (blue). Middle: Aerosol albedo at $405 \mathrm{~nm}$ (purple line), calculated from PAS and CRD data, and aerosol albedo at $450 \mathrm{~nm}$ (red triangles) measured by CAPS-ssa. Bottom: Aerosol absorbance measured by PAS at $405 \mathrm{~nm}$ (blue) and $530 \mathrm{~nm}$ (green), and estimated glyoxal concentrations in the chamber (red, calculated using wall loss rate $=2.7 \times 10-3 \mathrm{~s}-1$ onto cleaned Tedlar). Right axes are color-coded . 


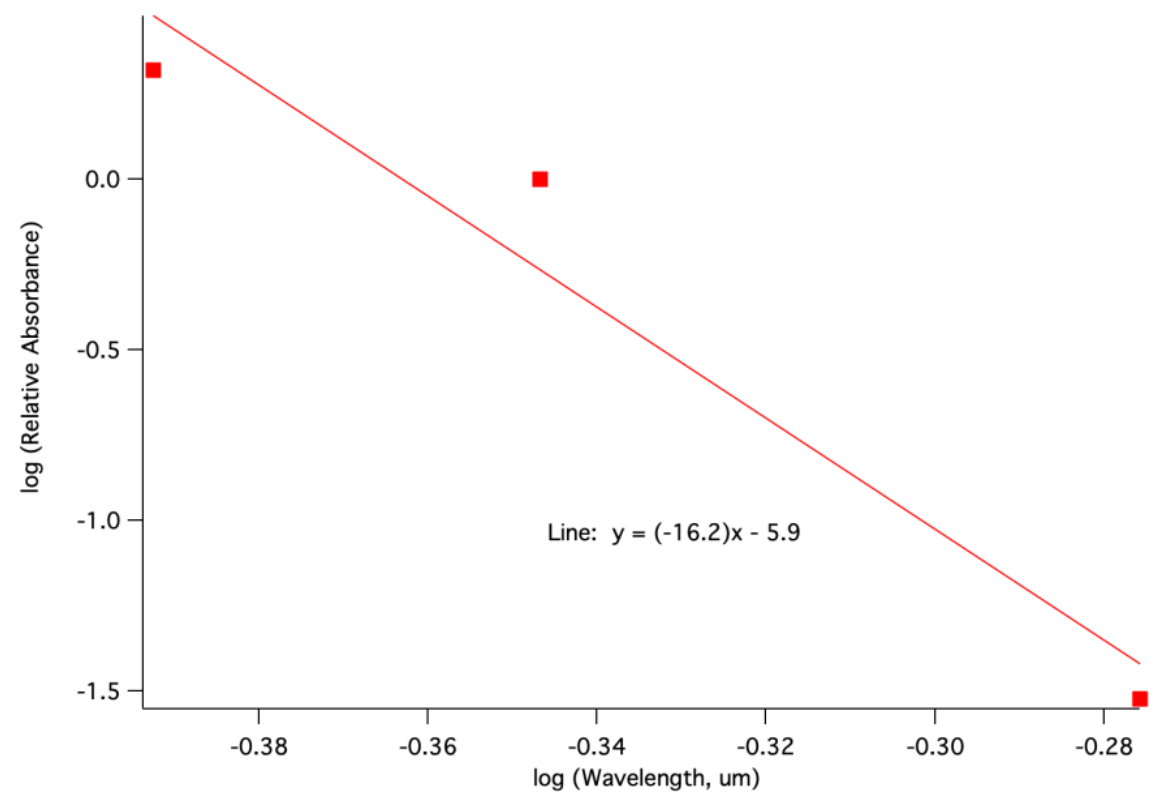

Figure S7: Calculation of angstrom absorption coefficient from normalized aerosol optical data recorded at 405, 450, and $530 \mathrm{~nm}$ at 2:20 pm in experiment 4 (300 ppb glyoxal + dry AS aerosol). Slope of line is Ångstrom absorption coefficient. 


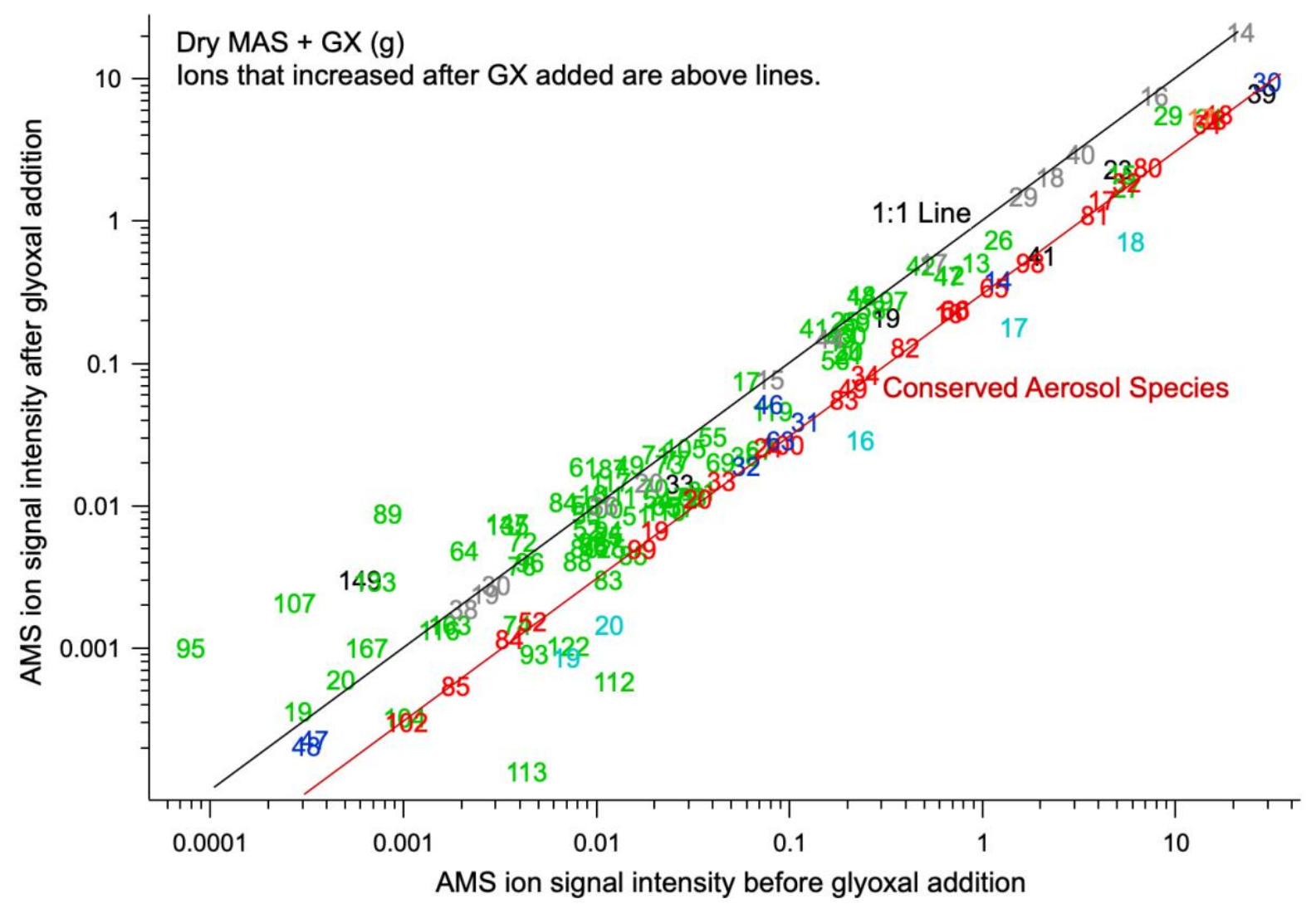

Figure S8: Experiment 8 AMS data summary: dry $\mathrm{MeAm}_{2} \mathrm{SO}_{4}$ aerosol +140 ppb glyoxal gas. Overall, aerosol concentrations decreased due to wall losses during the experiment, resulting in ions corresponding to conserved aerosol species falling on the red line with a slope of 1 , slightly below the 1:1 line. However, certain ions increased upon GX addition and appear above the red line: $\quad m / z 29(\mathrm{GX}$ fragment $\mathrm{CHO}), 26(\mathrm{CN}), 42$ and 97 (dimethylimidazole), among many others, while water peaks decreased at $m / z 16,17$, and 18 . 


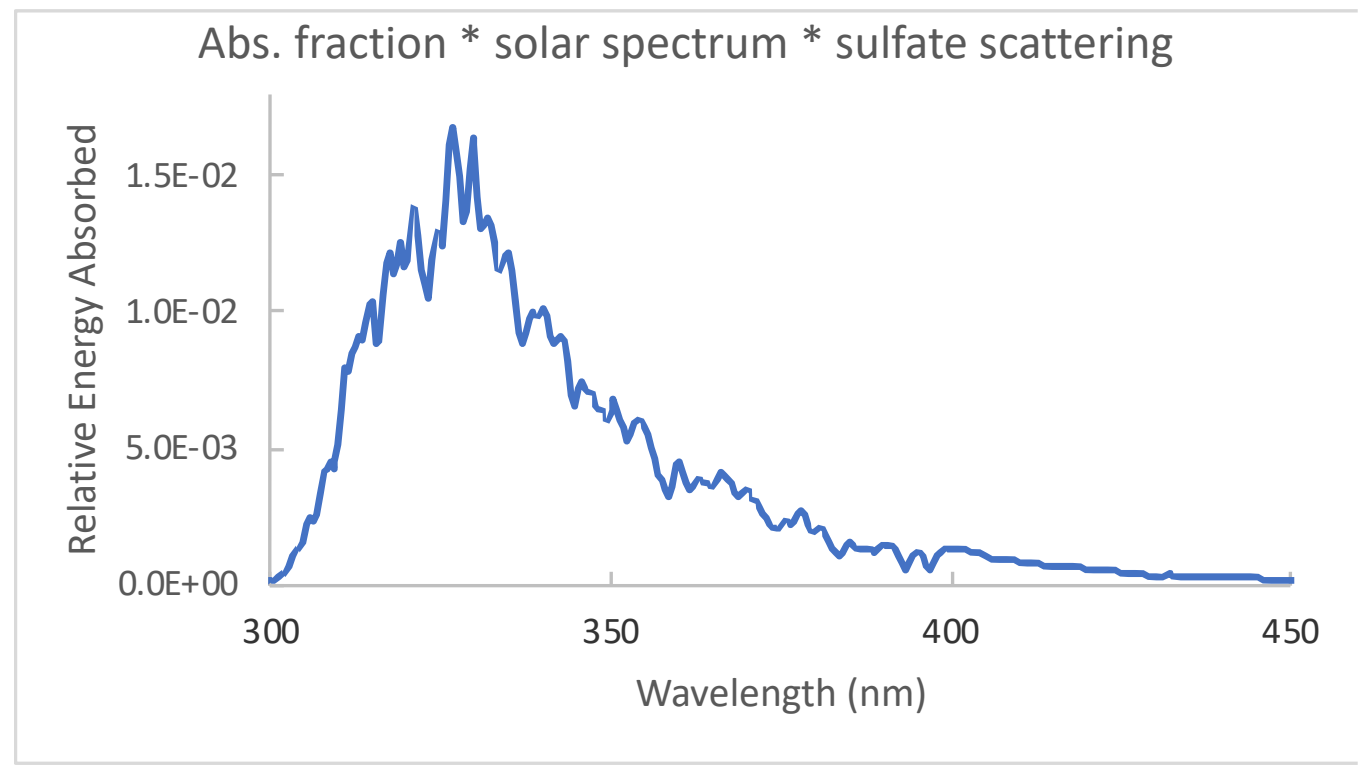

Figure S9: The relative energy absorbed by glyoxal + AS “dry" brown carbon is the calculated product of brown carbon absorbance fraction spectrum (using measured Ångstrom absorbance coefficient $=-16)$, ASTM G173-03 standard solar spectrum, and the AS aerosol scattering function. 1 Approximately $97 \%$ of the energy absorbed by this brown carbon is in the UV range $(<$ $400 \mathrm{~nm})$.

\section{References}

1. Nemesure, S.; Wagener, R.; Schwartz, S. E., Direct shortwave forcing of climate by the anthropogenic sulfate aerosol: Sensitivity to particle size, composition, and relative humidity. Journal of Geophysical Research: Atmospheres 1995, 100, (D12), 26105-26116. doi:10.1029/95JD02897 